\title{
Suicidal Behavior and Psychotropic Medication
}

\author{
Accepted as a Consensus Statement by the ACNP \\ Council, March 2, 1992
}

Depressed patients suffer from an illness that is common, involving about 11 million persons in the United States each year (Regier et al. 1990), and is associated with significant morbidity and mortality as a result of suicidal acts (Pokorny 1983; Beck et al. 1985). At the same time, patients are often reluctant to take medication because of a sense of hopelessness associated with the illness and a general failure to understand that depression is an illness needing treatment as opposed to personal weakness or a normal reaction to stress. For these and other reasons, it has been estimated that fewer than one third of the population suffering from a depressive illness seek treatment (Robins and Kulbok 1988). In this context, recent publicity in the broadcast and print media regarding claims that the antidepressant fluoxetine may trigger emergent suicidal and homicidal ideation and behavior in patients is of considerable importance. A depressive illness is the diagnosis most frequently associated with suicides ( $40 \%$ to $80 \%$ of cases; Pokorny 1983). Choosing the safest and most effective antidepressant for the depressed patient iscritical. Addressing the basis for doubts in the minds of clinicians and patients regarding the efficacy and safety of antidepressants is essential to avoid discouraging active treatment of depressed patients requiring it. Therefore, in July 1991, the American College of Neuropsychopharmacology charged a Task Force to review the scientificliterature for evidence regarding the effect of psychotropic medications on suicidality and to discuss what further research was needed as well as the implications for current clinical practice.

The members of the Task Force primarily involved in writing this report are: J. John Mann, M.D., Chair, Frederick K. Goodwin, M.D., Charles P. O'Brien, M.D., Ph.D., Donald S. Robinson, M.D.
The Task Force reviewed the available published scientific literature as well as unpublished data (supplied to regulatory agencies such as the Food and Drug Administration). In addition to data recently reviewed by the FDA Neuropsychopharmacology Review Committee, the Task Force also examined relevant unpublished data involving paroxetine, sertraline, and trazodone. The views presented in this position paper reflect those of the Council of the ACNP and the members of the Task Force.

\section{RATES OF SUICIDAL BEHAVIOR IN UNTREATED AND TREATED DEPRESSION}

Epidemiological data offer the opportunity to determine whether there are any large effects, either positive or negative, of treatment of medical disorders. In the instance of suicides complicating the treatment of depression, the study by Cassidy and Henry (1987) described results using a fatal toxicity index. The fatal toxicity index is a measure based on the rate of deaths due to "acute poisoning" per million National Health Service prescriptions issued for specific antidepressants during the years 1975 to 1984 in England, Wales, and Scotland. They also calculated indices for the number of standard units and gross quantities of antidepressant drugs prescribed. These three methods of calculation produced similar results. Deaths due to adverse reactions or multiple drugs were excluded. Concomitant alcohol intake was not an exclusion criterion. The fatal toxicity index for all antidepressant drugs was 34.9. Drugs introduced before 1970 had a significantly higher fatal toxicity index than drugs introduced after 1970. For example, for drugs introduced before 1970, the fa- 
tal toxicity indices were desipramine, 80.2; dothiepin, 50.0; amitriptyline, 46.5; nortriptyline, 39.2; doxipine, 31.3; imipramine, 28.4; and chlomipramine, 11.1. In contrast, for drugs released for marketing after 1970, the fatal toxicity index scores were much lower, with the exception of maprotiline. The scores were as follows: maprotiline, 37.6; trazodone, 13.6; valoxzine, 9.4; mianserin, 5.6; and nomifensine, 2.5. Thus, on the basis of suicide risk alone, regardless of the method of suicide, the newer generation, lower toxicity drugs appear to have a significant advantage. If one disregards the issue of emergent suicidal ideation or rates of suicide attempts and merely focuses on the risk of actual suicide, newer generation antidepressants do not appear to carry a greater risk than older generation antidepressants. Further comparable data are not yet available regarding the risk both of suicide attempts as well as completions for new serotonergic drugs, such as fluoxetine, fluvoxamine, and sertraline in the treatment of depression. Such data will be of importance in aiding physicians to choose the safest, most effective drugs for the more suicidal patient. The data regarding differential rates of suicide among antidepressants after controlling for prescription rates are not conclusive because it is conceivable that physicians prescribed older drugs to sicker, higher risk patients, or that side effects caused patients to take lower, subtherapeutic doses of older antidepressants.

In the United States there are no comparable national data bases on suicide or suicide attempt rates for each type of antidepressant or other kinds of psychotropic drugs, thereby making it very difficult to determine the risk for suicidal behavior associated with specific drugs. One approach to assessing the risk of suicidality in association with the use of fluoxetine for treatment of major depressive disorder is offered by a calculation that draws on the data base of the National Institute of Mental Health Epidemiologic Catchment Area (ECA) study (Regier et al. 1990). Although neither Eli Lilly and Company nor any other source can provide data on the indications for which fluoxetine is prescribed by physicians, one can conservatively estimate that some two thirds of fluoxetine prescriptions (i.e., for 2,000,000 out of the 3,000,000-plus prescriptions for the medication now being written annually) are for the treatment of depression. Analysis of ECA data (based on three sites) reveals that the annual rate of attempted suicide among persons with a concurrent major depressive disorder is $3.65 \%$. Applying this rate of "anticipated" instances of suicidal behaviors to $2,000,000$ persons - the estimated number of patients in 1990 year who took fluoxetine for treatment of severe depression-yields 73,000 instances of suicidal behavior.

In 1990, the total number of attempted suicides among patients with fluoxetine reported nationally via the FDA's Spontaneous Reporting System was 500. Thus, the number of reports of suicidal behavior was less than $0.7 \%$ of the number that would have been expected (i.e., 73,000) in the natural course of the disorder. Even if the FDA Spontaneous Reporting System were to pick up only 1 out of every 100 instances, suicdal behavior associated with the medication would still be only $68 \%$ of that expected in the natural course of the disorder. Thus, it appears that fluoxetine may suppress the rate of suicide attempts in the at-risk population receiving treatment with this drug.

Data supplied by the manufacturer of paroxetine indicate that in a population of 4,668 patients who were randomized to either paroxetine $(n=2963)$, placebo $(n=544)$, or other active antidepressants $(n=1,151)$ for 6-week double-blind control clinical trials and in some cases follow-up continuation pharmacotherapy or maintenance treatment studies, there were five sucides in the paroxetine group, two in the placebogroup, and three in the other active treatments group. When these data are converted to suicides per patient exposure year, the number of suicides per patient exposure year was 0.005 in the paroxetine group, 0.028 in the placebo group and 0.014 in the active control treatments group. There were no statistically significant differences across the three treatment groups. Withregard to attempted suicide there were also no significant differences among the three groups; the number of sucide attempts per patient exposure year was 0.40 in the paroxetine group, 0.083 in the placebo group, and .055 in the other active treatments group.

Data supplied to the authors by the manufacturer of sertraline are based on a population of 3,902 patients who were randomized to either sertraline $(n=1,938)$, placebo $(n=1,042)$, or other active antidepressants $(n=$ 922) for approximately 8 weeks of double-blind controlled clinical trials and, in some cases, follow-up continuation pharmacotherapy. There were six suicideattempts in the sertraline group $(0.3 \%)$, three in the placebo group $(0.3 \%)$, and two in the other active medications group $(0.2 \%)$, a result that was not statistically different. The frequencies of emergence of suicidal ideation and worsening of suicidal ideation were also examined in the various treatment groups. No significant differences were found among the groups in measures of suicide attempt rate, emergence of suicidal ideation (item 3 of the Hamilton Depression Scale; HAM-D) or worsening of suicidal ideation. Measures of change in severity of suicidal ideation (HAM-D item 3 scores from pretreatment to last visit) showed significantly greater improvement for the sertraline-treated patients compared with patients on placebo. Similar results were found with fluoxetine, trazodone, and paroxetine, as described below. 


\section{GROUP DATA ON THE OVERALL EFFICACY OF PSYCHOTROPIC AGENTS IN DEPRESSION AND OTHER CONDITIONS AND EMERGENT SUICIDAL BEHAVIOR OR IDEATION}

To detect emergent suicidal ideation from existing data sets, most investigators have utilized item 3 of the HAM-D, where a change from 0 ("absent") or 1 ("feels life is not worth living") to a score of 3 ("suicidal ideas or gesture") or 4 ("attempts at suicide") has been a generally used definition. Some analyses have assessed emergent suicidal ideation at any point during a 6-week double-blind study. Others have employed an endpoint analysis. This distinction does not appear critical because overall, analyses of data have generated similar results using either approach. Another approach employed in the analyses of the paroxetine data involved examining rates of emergent suicidal ideation in patients without suicidal ideation at baseline. This interesting strategy revealed that the rate of newly emergent suicidality was higher in the placebo group than either the paroxetine or the active treatment group. This finding indicates the potential for newly emergent suicidal ideation in untreated depressed patients who do not report such ideation at the start of treatment.

A meta analysis of double-blind controlled studies involving 3,065 patients who received either fluoxetine, a reference antidepressant, or placebo found a greater degree of improvement in item 3 of the HAM-D in the fluoxetine and active drug groups compared with placebo (Beasley et al. 1991). Moreover, emergent suicidality, as defined by a change from $0-1$ at baseline to 34 on item 3 of the HAM-D scale, occurred with significantly lower frequency in the fluoxetine and reference drug groups than on the placebo group. Two other double-blind controlled antidepressant treatment studies found the effect of fluoxetine on suicidality ratings to be superior to mianserin (Muijen et al. 1988) and comparable to amitriptyline (Altamura et al. 1989), respectively. An interesting study by Sacchetti and colleagues (1991) found that depressed patients with a history of suicidal behavior had a superior response to fluoxetine compared with desipramine or nortriptyline in a double-blind study. Four of six double-blind studies comparing a selective serotonin reuptake inhibitor to a reference antidepressant found an initial advantage for the serotonin drug in terms of early (up to 4 weeks of treatment) improvement in suicidal ideation (Montgomery et al. 1981; de Wilde et al. 1991; Mullin et al. 1988; Gonella et al. 1990; Nathan et al. 1990; Perez and Ashford 1990). This difference in treatment response was no longer significant by 6 weeks of treatment.

A retrospective analysis of the data (published and unpublished) from all placebo-controlled trials of trazodone (Desyrel) included in its NDA comparing it with tricyclic antidepressants (either imipramine or amitriptyline) and placebo reveals no evidence of emergence of suicidal ideation or actual suicide attempts during active drug treatments compared with placebo treatment. Also, there was no difference in HAM-D item 3 (suicidal ideation) improvement ratings between trazodone and tricyclic treatment (DS Robinson, personal communication). In a study of 341 hospitalized patients with endogenous depression included in the NDA, $8 \%$ of 120 trazodone-treated patients reported significant suicidal ideation (HAM-D item 3 score of 3 or 4 ) at end of treatment compared with $11 \%$ of the imipramine- and $20 \%$ of the placebo-treated patients. In this study overall group improvement in suicidal ideation was significantly greater with trazodone and imipramine than with placebo treatment.

Since its market introduction in March 1982, at least $10,000,000$ patients in the United States have been treated with trazodone. During this time, there have been a total of only 56 spontaneous adverse reports of deaths occurring during Desyrel treatment, with 20 of these classified as probable suicides. Given a national yearly suicide rate of $12 / 100,000$, the rate of known suicides involving patients receiving trazodone is only about one sixth of the rate in the general population. The rate of suicide in depressed patients (the commonest indication for trazodone treatment) is about 40to 60 -fold greater than in the general population, and therefore it is likely that trazodone reduces the suicide rate. It is reasonable to assume that this beneficial effect is characteristic of antidepressants in general.

The tricyclic antidepressant maprotiline was tested for its efficacy in preventing relapse into depression by a group of French investigators (Rouillon et al. 1989). They studied 1,141 who had been treated successfully for major depression. Patients were randomly assigned to either low-dose maprotiline $(75 \mathrm{mg} ; n=385)$, verylow-dose maprotiline $(37.5 \mathrm{mg}, n=382)$, or placebo ( $n=374)$. During the 1-year follow-up, low-dose maprotiline was significantly superior to placebo in preventing relapse ( $16 \%$ vs. $37.5 \%)$. However, among those relapsing, there was no difference in suicide rate between the maprotiline groups and those receiving placebo. The suicide attempt rate for the placebo group was significantly lower than the rate for those patients receiving maprotiline, but all three groups had rates of suicide attempts lower than those previously reported during bouts of depression (Avery and Winokur 1978). The report of this study failed to give any information about the proportion of patients in each treatment group who had previously attempted suicide, a variable which has an important bearing on the results because a history of suicide attempts predicts future attempts. Thus, this study shows a dissociation between antidepressant efficacy and frequency of suicide attempts. The study 
does not directly address the issue of whether the antidepressant was causally related to suicide attempts or whether the placebo group simply had a lower than expected suicide attempt rate for a population of depressed patients.

\section{CASE REPORTS OF EMERGENT SUICIDALITY}

This section will focus on case reports of emergent suicidal behavior in patients who have received fluoxetine as well as other agents. There have been at least four published case reports of emergent suicidality involving mostly ideation but also actual attempts in a total of nine patients receiving fluoxetine for depression (Dasgupta, 1990; Teicher et al. 1990; Hoover, 1991; Masand et al. 1991) and six patients with obsessive compulsive disorder (King et al. 1991). One case report (Hoover, 1990) was subsequently retracted because the same patient developed the same kind of emergent suicidality on imipramine as had originally been seen and reported while on fluoxetine (Hoover 1991). These cases are difficult to interpret because there is no consistent time interval on fluoxetine before the emergence of significant new suicidal ideation or behavior and there is no consistent dose level at which suicidality emerges. Moreover, a significant proportion of these cases had such symptoms and a history of suicide attempts in the past; the best predictor of a future suicide attempt is a history of suicide attempt, emphasizing the importance of a control group treated by another class of antidepressant. These cases cannot distinguish the natural course of the illness, the impact of external events, and the potential effects of the treatment. Fava and Rosenbaum (1991) surveyed a number of physicians who reported on their experience in treating 1,017 depressed patients with a variety of antidepressants. They reported no difference in the rate of emergent suicidality in the group treated with fluoxetine alone compared with all other patients. A reanalysis of their data comparing all fluoxetine-treated cases with all nonfluoxetine-treated cases indicated a higher rate of emergent suicidality in the fluoxetine group. Fava and Rosenbaum (1991) state that their survey was conducted before the publicity generated by the Teicher et al. (1990) study that reported a link between fluoxetine and emergent suicidality; nevertheless, their study was not double blind and the results are far from conclusive.

A review of the literature indicates that reports of emergent suicidality or aggressivity have involved a wide range of pharmacologically diverse antidepressants, including imipramine (Tec 1963; Gottschalk et al. 1965; Rampling 1978); amitriptyline (Rampling 1978; Soloff et al. 1986; Soloff et al. 1987); desipramine (Damluji et al. 1988); nortriptyline (Damluji et al. 1988); amoxapine (Damluji et al. 1988); trazodone (Damluji and Fer- guson 1988); alprazolam (Montgomery 1988); and maprotiline (Rouillon et al. 1989). The patients involved in the case reports for each drug ranged from as few as one to as many as five. The diagnoses were also diverse and included depression, somnambulism, narcolepsy, cataplexy, and borderline personality disorder.

There have been individual case reports in the literature describing aggressive behavior in association with the use of various antidepressant medications, antianxety medications, and neuroleptic medications. Several authors (Ingram and Timbury 1960; DiMascio et al. 1969; DiMascio et al. 1970; Hall and Zisook 1981) have reported that benzodiazepine medications produce hostility and occasional paradoxical rage responses. Such rage reactions appear to be rare because they are not found even when these medications are administered to prisoners prone to violence. No data are available that provide information about the frequency of these events. If they are indeed related to benzodiazepines, this effect only occurs in specifically susceptible individuals.

With respect to neuroleptic medications, a common side effect is akathisia (severe restlessness). This restlessness or agitation is more frequent with high-dose, high-potency neuroleptics, and it has been suggested that this side effect can increase the likelihood of violent or suicidal behavior. A possible causal relationship between neuroleptic-induced akathisia and violence has been suggested in several case reports and at least one organized study (Shear et al. 1983; Siris 1985; Herrera et al. 1988). In contrast, numerous other studies have shown that neuroleptics can be useful in controlling the violent behavior sometimes seen in psychotic patients. At present, there are insufficient data to make a frrm conclusion, but it would appear that, as with all drugs, there are individual differences in patient response. Thus, while high-potency neuroleptics are helpful in controlling violent behavior, in some patients the akathisia side effect may aggravate or provoke aggression or suicidality.

In conclusion, case reports suggest that a small minority of patients may experience emergent suicidal thoughts or evince such behavior during the pharmacological treatment of depression. These reports do not distinguish between the relative potential contribution of the disease process, external stressors, or the medication. Of significance, there is evidence that such emergent suicidality is not specific to any one type of antidepressant and may therefore be largely a manifestation of the natural course of the illness.

\section{SCIENTIFIC ISSUES}

There are several possible approaches to assessing the association of antidepressant drug treatment and surcidality (suicidal acts and ideation). One approach is 
to perform meta analyses of pooled data from doubleblind clinical trials of patients with major depressive disorder comparing the incidence of suicides (and attempted suicides) among the patient groups treated with the index antidepressant, the comparative antidepressant, and placebo. A major problem in the United

relative rates of suicide attempts and completions in patients treated with specific antidepressants. Controlled treatment studies of depressed patients tend to exclude very suicidal patients and only include a selected segment of the depressed patient population. Thus, although analyses of such data sets are useful, they do involve a lower risk population.

Suicidal ideation (usingitem 3 of the HAM-D) could be assessed over the course of double-blind treatment. Emergence of suicidal ideation has typically been defined as a change in the HAM-D item 3 from 0 to 1 at baseline, to 3 or 4 during the clinical trial, or alternatively, any worsening of the HAM-D item 3 in patients with a baseline rating of 0 (no ideation). One such study has been published recently with regard to fluoxetine (Beasley et al. 1991). However, the rate of emergent suicidal ideation may only be weakly proportional to the risk for suicide attempts or completion.

A second approach would be to estimate the incidence of a relatively rare event (suicide in association with antidepressant use) by conducting an epidemiological study employing a large data base (such as statewide medicaid and private health care systems). The study would describe the profiles of age-, sex-, and data-matched cohorts for the index antidepressant, a standard antidepressant drug, and control populations of patients in each data set. The demographics, morbidity, and drug exposure profile would be tabulated. The relative risk of death from all causes, including suicide, would be determined in the three patient groups (index antidepressant, comparative antidepressant, and control) based on surveillance over 60, 180 and 365 days following initiation of therapy (or, for controls, matched data).

A third approach is to conduct case-controlled studies utilizing patients at low risk for suicide and treated with an antidepressant for another clinical indic

by primary care physicians for weight control and treatment of compulsive eating. Fluoxetine is currently being investigated as an antiobesity medication. The relative death rates, incidence of suicides, and self-destructive behavior in these fluoxetine-treated patients could be compared with the placebo control group. The incidence of suicidal ideation may be difficult to determine in these studies because of the lack of data from an appropriate depression rating instrument such as the HAM-D.

A fourth possible approach is to design a study employing an enriched sample of patients who have a high risk of suicide and self-destructive behaviors. A parallelgroup design could be employed to compare the incidence of suicidal behavior in this special patient population treated in a double-blind parallel group design study with either a serotonin reuptake inhibitor, a tricyclic antidepressant, or placebo.

Claims that serotonin-enhancing drugs may generate an increase in suicidal ideation, aggressive behavior, or actual suicidal acts are paradoxical in the face of a large body of data indicating that enhancement of serotonergic activity should tend to raise the threshold for suicidal and aggressive behavior. It should be emphasized that the suggestions that such adverse effects are triggered by drugs such as fluoxetine are currently speculative and unproven. Therefore, we are discussing the potential basis for what is, at present, a hypothetical event. It has been suggested that selective serotonin inhibitors work by increasing exposure of $5-\mathrm{HT}_{1 \mathrm{~A}}$ receptors to serotonin (Blier et al. 1990). The goal is to increase transmission at $5-\mathrm{HT}_{1 \mathrm{~A}}$ receptors at the serotonin terminal fields. There may be little or no initial increase in serotonin transmission until the presynaptic somatodendritic $5-\mathrm{HT}_{1 \mathrm{~A}}$ autoreceptors on the cell bodies are desensitized. Postsynaptic 5- $\mathrm{HT}_{1 \mathrm{~A}}$ receptors resist desensitization, resulting in a progressive increase in transmission because desensitization of the autoreceptors will increase the level of serotonin release.

One possible mechanism that could reconcile the hypothesis that less serotonin is associated with a lower threshold for suicidal behavior and an increased probability of suicide, with the suggestion that fluoxetine may be associated with paradoxical increased suicidability in vulnerable patients, is as follows: a serotonin reuptake inhibitor may initially activate $5-\mathrm{HT}_{1 \mathrm{~A}}$ somatodendritic autoreceptors and, because of failure to desensitize, continue to inhibit serotonin release, resulting in a prolonged decrease in overall serotonin transmission instead of a progressive increase in transmission.

\section{CLINICAL ISSUES AND CONCLUSIONS}

1. Antidepressants are effective treatments for depression, and, in the vast majority of patients, result in substantial improvement or total remission of suicidal ideation and impulses.

2. New-generation low-toxicity antidepressants, including selective serotonin reuptake inhibitors, may carry a lower risk for suicide than older tricyclic antidepressants. This possible safety advantage may be a consequence of lower toxicity in the event of a drug overdose. This consideration should be balanced by the clinician's judgement regarding potential efficacy for each individual patient. The 
choice of treatment is based on a drug's relative efficacy as well as safety.

3. There is no evidence that antidepressants such as the selective serotonin reuptake inhibitors, for example, fluoxetine, trigger emergent suicidal ideation over and above rates that may be associated with depression and other antidepressants. What is clear is that most patients receive substantial benefit from treatment with this drug and related antidepressants.

4. It is good clinical practice to monitor patients receiving treatment with antidepressants with regard to general clinical progress, and in particular, suicidal ideation and impulses. It is well known that depressed patients who are responding to treatment may occasionally have brief relapses lasting one or two days in which their symptoms may recur. Side effects such as akathisia may be associated with a worsening psychiatric state. Patients should be warned that suicidal ideation may occasionally worsen in the course of treatment, as may overall depression, and that such an event would be a reason for immediately contacting their doctor. Applying this standard clinical practice to all patients would constitute a reasonable safeguard in the event that there are, indeed, a small minority of vulnerable patients who are at risk for emergent suicidal ideation. It should be recognized that such emergent suicidal ideation may be the consequence of the patient's illness, adverse changes in the life situation, or perhaps, in a few cases, because of an adverse effect of the antidepressant.

\section{REFERENCES}

Altamura AC, Percudani M, Guercetti G, Invernizzi G (1989): Efficacy and tolerability of fluoxetine in the elderly: A double-blind study versus amitryptiline. Int Clin Psychopharmacol 4(suppl 1):103-106

Avery D, Winokur G (1978): Suicide, attempted suicide, and relapse rates in depression. Arch Gen Psychiatry 35:749-753

Beasley CM, Dornseif BE, Bosomworth JC, Sayler ME, Rampey AH, Heiligenstein JH, Roberts VL, Murphy DJ, Mascia DN (1991): Fluoxetine and suicidality: Absence of association in controlled depression trials. Br Med J 303:685-692

Beck A, Steer RA, Kovacs M, Garrison B (1985): Hopelessness and eventual suicide: A 10-year prospective study of patients hospitalized with suicidal ideation. Am J Psychiatry 142:559-563

Blier P, Montigny C, Chaput Y (1990): A role for the serotonergic system in the mechanism of action of antidepressant treatments: Preclinical evidence. J Clin Psychiatry 51(suppl 4):14-21

Cassidy and Henry (1987): Fatal toxicity of antidepressant drugs in overdose. Br Med J 295:1021-1024
Damluji NF, Ferguson JM (1988): Paradoxical worsening of depressive symptomatology caused by antidepressants. J Clin Psychopharmacol 5:347-349

Dasgupta K (1990): Additional cases of suicidal ideation associated with fluoxetine. Am J Psychiatry 147:1570

DiMascio A, Shader RI, Harmatz J (1969): Psychotropic drugs and induced hostility. Psychosomatics 10:46-47

DiMascio A, Shader RI, Harmatz JS (1970): Behavioral toxic. ity, V: Gross behavior patterns. In Shader RI, DiMascio A (eds), Psychotropic Drug SideEffects: Clinical and Theoretical Perspectives. Philadelphia, Williams \& Wilkins, pp 142-148

Fava M, Rosenbaum JF (1991): Suicidality and fluoxetine: Is there a relationship? J Clin Psychiatry 52:108-111

Gonella G, Baignoli G, Ecari U (1990): Fluvoxamine and im. ipramine in the treatment of depressive patients: $A$ double-blind controlled study. Cur Med Res Opin 12: 177-184

Gottschalk LA, Gleser GC, Wylie HW, Jr., Kaplan SM(1965): Effect of imipramine on anxiety and hostility levels. Psychopharmacologia 7:303-310

Hall RCW, Zisook S (1981): Paradoxical reactions to benzodiazepines. Br J Clin Pharmacol 11:99S-104S

Herrera JN, Sramek JJ, Costa JF, Roy S, Heh CW, Nguyen BN (1988): High potency neuroleptics and violence in schizophrenics. J Nerv Ment Dis 176:558-561

Hoover CE (1990): Additional cases of suicidal ideation associated with Fluoxetine (Letter). Am J Psychiatry 147:1570-1571

Hoover CE (1991): Suicidal ideation not associated with fluoxetine. Am J Psychiatry 148:543

Ingram IM, Timbury GC (1960): Side effects of librium. Lancet 2:766

King RA, Riddle MA, Chappell PB, Hardin MT, Anderson GM, Lombroso P, Scahill L (1991): Emergence of selfdestructive phenomena in children and adolescents during fluoxetine treatment. J Am Acad Child Adolesc Psychiatr 30:179-186

MasandP, Gupta S, Dewan M (1991): Suicidal ideation related to fluoxetine treatment. N Engl J Med 324:420

Montgomery SA (1988): The benefits and risks of 5-HT uptake inhibitors in depression. Br J Psychiatry 153:7-10

Montgomery SA, McAuley R, Rani SJ, Roy D, Montgomery DB (1981): A double blind comparison of zimelidine and amitriptyline in endogenous depression. Acta Psychiatr Scand 290(suppl):314-327

Muijen M, Silverstone T, Mehmet A, Christie M (1988): A comparative clinical trial of fluoxetine, mianserin and placebo in depressed outpatients. Acta Psychiatr Scand 78:384-390

Mullin JM, Pandita-Gunawardena VR, Whitehead AM (1988): A double-blind comparison of fluvoxamine and duthiepin in the treatment of major affective disorder. Br J Clin Pract 42:51

Nathan RS, Perel JM, Pollock BG, Kupfer DJ (1990): The role of neuropharmacologic selectivity in antidepressant action: Fluvoxamine versus desipramine. J Clin Psychiatry $51: 367-372$

Perez A, Ashford JJ (1990): A double-blind, randomized comparison of fluvoxamine with mianserin in depressiveillness. Curr Med Res Opin 12:234 
Pokorny AD (1983): Prediction of suicide in psychiatric patients: A report of a prospective study. Arch Gen Psychiatry 40:249-257

Rampling D (1978): Aggression: A paradoxical response to tricyclic antidepressants. Am J Psychiatry 135:117-118

Regier DA, Farmer ME, Rae DS, Locke BZ, Keith SJ, Judd LL, Goodwin FK (1990): Comorbidity of mental disorders with alcohol and other drug abuse. JAMA 264:2511-2518

Robins LN, Kulbok PA (1988): Epidemiological studies in suicide. Psychiatric Annals 18:619-627

Rouillon F, Phillips R, Serrurier D, AnsartE, Gerard MJ (1989): Réchutes de depression unipolaire et éfficacité de la maprotiline. L'Encéphale XV:527-534

Sacchetti E, Vita A, Guarneri L, Cornarcchia M (1991): The effectiveness of fluoxetine, Clomipramine, nortriptyline, and desipramine in major depressives with suicidal behavior: Preliminary findings. In Cassano GB, Akiskal HS (eds), Serotonin related psychiatric syndromes: Clinical and therapeutic links, Royal Society of Medicine Services Limited, London
Shear MK, Frances A, Weiden P (1983): Suicide associated with akathisia and depot fluphenazine treatment. J Clin Psychopharmacol 3:235-236

Siris SG (1985): Three cases of akathisia and "acting out." J Clin Psychiatry 46:395-397

Soloff PH, George A, Nathan RS, Schulz PM, Perel JM (1986): Paradoxical effects of amitriptyline on borderline patients. Am J Psychiatry 143:1603-1605

Soloff PH, George A, Nathan RS, Schulz PM, Perel JM (1987): Behavioral dyscontrol in borderline patients treated with amitriptyline. Psychopharmacol Bull 23:177-181

Tec L (1963): Unexpected effects in children treated with imipramine. Am J Psychiatry 120:603

Teicher MH, Glod C, Cole JO (1990): Emergence of intense suicidal preoccupation during fluoxetine treatment. Am J Psychiatry 147:207-210

de Wilde J, Mertens C, Fredricson Overo K, Hepfner Peterson HE (1991): Citalopram versus mianserin. Acta Psychiatr Scand 1985:89-96 